\title{
A Pilot Study of Once-Daily Antiretroviral Therapy Integrated With Tuberculosis Directly Observed Therapy in a Resource-Limited Setting
}

\author{
Christopher Jack, MBChB, * Umesh Lalloo, MBChB, MD, * Quarraisha Abdool Karim, PhD, * \\ Salim Abdool Karim, MBChB, PhD, * Wafaa El-Sadr, MD, MPH, † Sharon Cassol, PhD, * $\neq$ and \\ Gerald Friedland, $M D \S$
}

\begin{abstract}
Summary: To determine the feasibility and effectiveness of integrating highly active antiretroviral therapy (HAART) into existing tuberculosis directly observed therapy (TB/DOT) programs, we performed a pilot study in an urban TB clinic in South Africa. Patients with smear-positive pulmonary TB were offered HIV counseling and testing. Twenty HIV-positive patients received once-daily didanosine (400 mg) plus lamivudine (300 mg) plus efavirenz (600 mg) administered concomitantly with standard TB therapy Monday to Friday and self-administered on weekends. After completing TB therapy, patients were referred to an HIV clinic for continued treatment. At baseline, patients had a mean CD4 count of 230 cells $/ \mathrm{mm}^{3}$ (range: 24-499 cells $/ \mathrm{mm}^{3}$ ) and a mean viral load of $5.75 \log _{10}$ (range: $3.81-$ $7.53 \log _{10}$ ). Seventeen completed combined standard TB and HIV therapy; 16 of $20(80 \%)$ patients enrolled and 15 of $17(88 \%)$ patients completing standard TB therapy achieved a viral load $<50$ copies $/ \mathrm{mL}$ and mean CD4 count increase of 148 cells $/ \mathrm{mm}^{3}$. TB was cured in 17 of $20(85 \%)$ enrolled patients and 17 of $19(89 \%)$ patients with drugsensitive TB. Treatment was well tolerated, with minimal gastrointestinal, hepatic, skin, or neurologic toxicity. The project was well accepted and integrated into the daily TB clinic functions. This pilot study demonstrates that TB/DOT programs can be feasible and effective sites for HIV identification and the introduction and monitoring of a once-daily HAART regimen in resource-limited settings.
\end{abstract}

Key Words: HIV/AIDS, HAART, access to antiretroviral therapy, tuberculosis directly observed therapy, once-daily HAART

(J Acquir Immune Defic Syndr 2004;36:929-934)

Received for publication November 19, 2003; accepted April 15, 2004.

From the *Nelson R. Mandela School of Medicine, University of Kwa Zulu Natal, Durban, South Africa; †Columbia University Mailman School of Public Health and Harlem Hospital, New York, NY; $\$$ Africa Centre for Health and Population Research, Durban, South Africa, and §AIDS program, Yale University School of Medicine, New Haven, CT.

Supported by grants from the Doris Duke Charitable Foundation, Irene Diamond Fund, and Wellcome Trust.

Reprints: Gerald H. Friedland, Yale University School of Medicine, AIDS Program, 135 College Street; Suite 323, New Haven, CT 06520 (e-mail: Gerald.Friedland@yale.edu).

Copyright $(2004$ by Lippincott Williams \& Wilkins
T uberculosis (TB) is a major cause of morbidity and mortality among persons with HIV disease worldwide, particularly in resource-poor settings. The province of KwaZulu Natal is the most populous of the 9 provinces in South Africa and is most severely affected by the HIV pandemic. ${ }^{1,2}$ As in many resource-limited settings, the HIV epidemic in KwaZulu Natal is accompanied by a severe TB epidemic. Approximately two thirds of TB patients are coinfected with HIV, which results in a high annual case fatality rate of approximately $40 \%{ }^{2}$

Highly active antiretroviral therapy (HAART) has dramatically altered the natural history of HIV disease in industrialized countries. ${ }^{3}$ High cost, weak infrastructure, and lack of political will have limited HAART availability in resourceconstrained settings such as KwaZulu Natal, however. ${ }^{4}$ The existence of TB directly observed therapy (DOT) programs and the recent availability of less costly, safer, and more convenient HAART regimens provide a unique but as yet untested opportunity to integrate the treatment of TB and HIV disease. This is particularly true because TB/DOT programs are designed to enhance and support medication adherence, which is crucial for therapeutic success for both diseases. Treating TB and HIV disease concomitantly may potentially improve the outcome for each. Challenges to implementing such a strategy include (1) disruption and overburdening of TB health care services, (2) excessive cost, (3) overlapping and additive drug interactions and toxicities, ${ }^{5,6}(4)$ paradoxic and immune reconstitution syndromes, ${ }^{7}$ and (5) potential decreased program and medication adherence. ${ }^{8}$ To begin to address these issues, we conducted a pilot feasibility study integrating HAART into an existing TB/DOT program in an urban metropolitan public TB clinic, the Prince Cyril Zulu Communicable Diseases Clinic in Durban, KwaZulu Natal, South Africa.

\section{MATERIALS AND METHODS}

\section{Setting}

The Prince Cyril Zulu Communicable Diseases Clinic is a public municipal clinic providing TB diagnosis and treat- 
ment for the metropolitan area of Durban, KwaZulu Natal. DOT is provided on-site or through referral to community care settings once the diagnosis of TB is established.

\section{Patients}

Eligibility criteria included sputum smear-positive pulmonary TB, confirmed HIV infection, 18 years of age or greater, receipt of standard TB therapy, resident of the greater Durban area, and ability to attend the TB/DOT program at the Cyril Zulu Communicable Diseases Clinic daily. Pregnant and breast-feeding women and those not able to practice reliable contraception were excluded, as were patients with baseline liver enzymes $>5$ times the upper limit of normal, a history of pancreatitis, significant peripheral neuropathy, prior antiretroviral treatment, and a CD4 cell count $>500$ cells $/ \mathrm{mm}^{3}$. Patients provided written informed consent.

\section{Study Procedures}

HIV voluntary counseling and testing (Vironostika HIV-1/HIV-2, Organon Teknika; Murex HIV 1+2 GAC, Murex Wellcozyme) were offered to consecutive patients with sputum smear-positive pulmonary TB. Consecutive patients found to be HIV-positive were offered study participation, and after providing written informed consent, they were screened for study eligibility. Those meeting eligibility requirements were enrolled.

The study was designed to employ standard procedures, practices, and definitions used by the clinic and recommended by the South Africa National Tuberculosis Control Program. ${ }^{9}$ Patients received standard TB treatment as per the clinic protocol, consisting of an intensive phase of rifampicin, isoniazid, pyrazinamide, and ethambutol in single-combination tablets according to a subject's weight for 2 months. TB drugs were administered under direct observation from Monday to Friday. After 2 months of the intensive phase of TB treatment, 2 drugs (rifampicin and isoniazid) in single combination tablets were administered in a continuation phase daily from Monday to Friday for the remaining treatment period, which was usually 4 months. Management and assessment of the response to TB treatment were carried out according to the South African National Tuberculosis Control Program Guidelines. ${ }^{9}$ Patients identified as HIV-positive but not meeting the study entry criteria were offered referral to the HIV Clinic at the Nelson R. Mandela School of Medicine for further assessment and follow-up.

HAART was administered concomitantly with TB medications. Patients received and ingested their TB and HIV medications at the same time under direct observation by the clinic staff nurses. This was routinely done in the morning at about 9:00 AM. Two daily dose packs of HIV medication were provided on Friday for weekend self-administration at home. The regimen, consisting of once-daily administration of 400 $\mathrm{mg}$ of didanosine (ddI; in tablet formulation, dose adjusted to
$300 \mathrm{mg}$ if patient weight $<60 \mathrm{~kg}$ ), $300 \mathrm{mg}$ of lamivudine (3TC), and $600 \mathrm{mg}$ of efavirenz, was chosen on the basis of drug availability in South Africa at the time of ethical approval and existing data about pharmacologic properties, clinical efficacy, and potential interactions with TB medications. ${ }^{10-12}$

Assessments for evidence of drug toxicity and clinical efficacy were initially done weekly for the first 4 weeks and then monthly thereafter; they consisted of clinical evaluations and laboratory tests with particular attention to gastrointestinal, neurologic, dermatologic, and psychiatric adverse effects. CD4 cell counts (FACScan; Becton Dickinson) and viral load testing (Nuclisens HIV-1 QT) were done at baseline and every 2 months during concomitant treatment. HIV genotypic resistance was determined at baseline and at 6 months by means of plasma specimens obtained from patients with detectable virus (ViroSeq; Applied Biosystems) and was compared with reference strains in the Stanford HIV Database (http://hivdb. Stanford.Edu/hiv/).

Adverse events were assessed for severity using the AIDS Clinical Trials Group (ACTG)/Community Program for Clinical Research on AIDS (CPCRA) grading scheme (http://www.nih.gov). Each Monday morning, a standardized validated questionnaire adopted for local use was administered by study staff regarding weekend medication adherence. ${ }^{8}$ Social support was enhanced during the study and included ongoing counseling by clinic and research staff. Transportation costs were provided as needed. No financial incentives were provided.

Medical and nursing staff at the Cyril Zulu Clinic were trained in the study procedures and provided and observed medication administration as with other clinic patients. In addition, the research team consisted of a specialist physician on a $20 \%$ basis and a nurse and administrative assistant on a $50 \%$ basis each. On successful completion of anti-TB treatment, the patients were transferred to the Infectious Disease Clinic of the King Edward VIII Hospital, the teaching hospital of the Nelson R. Mandela School of Medicine, University of Kwa Zulu Natal, for continued antiretroviral therapy.

All patient materials were available in English and Zulu. Study data were collected on standardized case report forms. Results were analyzed for all patients who took 1 or more doses of HAART and for those completing the treatment protocol.

The Ethics Committee of the Nelson R. Mandela School of Medicine, University of Kwa Zulu Natal, and the Human Investigations Committee, Yale University School of Medicine, approved the study.

\section{RESULTS}

From January 2002 to June 2002, 92 consecutive patients with smear-positive pulmonary TB were counseled and tested for HIV and offered study participation. Of these, 59 (64\%) tested positive and 20 met entry criteria and were en- 
rolled in the study, of whom 15 were women and 5 were men, with a mean age of 31 years (range: $18-53$ years), a mean CD4 count of 230 cells $/ \mathrm{mm}^{3}$ (range: $24-499 \mathrm{~mm}^{3}$ ), and a mean viral load of $5.75 \log _{10}$ (range: $3.81-7.53 \log _{10}$ ). Of the remaining 39 patients who were not enrolled, 6 declined participation, 8 did not return for their results, 13 were referred to outlying clinics for TB treatment, 7 were unable to attend clinic daily for DOT, 3 had CD4 counts $>500$ cells $/ \mathrm{mm}^{3}$, and 2 did not meet the medical entry criteria.

Ten patients commenced HAART within 2 weeks of starting TB medication: 8 between 1 and 2 months and 2 after 2 months. Seventeen of the 20 enrolled patients completed standard TB therapy as defined by the South African National Tuberculosis Control Program. ${ }^{9}$ Two patients withdrew during the study, and 1 of these was lost to follow-up. One patient was retrospectively diagnosed as having multidrug-resistant TB at study enrollment and was hospitalized to receive secondline TB therapy and continue antiretroviral therapy.

Eighteen of 20 patients $(90 \%)$ reported an improvement in physical and mental health. Sixteen of the $20(80 \%)$ enrolled patients achieved a viral load $<50$ copies $/ \mathrm{mL}$ at 24 weeks (Figs. 1, 2), and 15 of 17 (88\%) patients who completed concomitant standard TB and HIV treatment achieved a viral load $<50$ copies/mL (see Figs. 1, 2). Among those who completed standard TB and HIV therapy, there was a mean increase of 148 CD 4 cells $/ \mathrm{mm}^{3}$ (range: $20-379$ cells $/ \mathrm{mm}^{3}$ ) (Fig. 3). Of the 2 patients on standard TB therapy and HAART who did not achieve complete viral suppression, 1 was nonadherent to HAART and the other was unexplained. Two patients without baseline resistance mutations were documented to have developed resistance at 24 weeks to 3TC, ddI, and efavirenz. Both patients developed V106M, Y181C, and G190S mutations. In addition, 1 patient developed K65R, and the other patient developed L74V, K101E, M184V, and R219N mutations.

TB cure as defined by the South African Tuberculosis Control Program ${ }^{9}$ was achieved over a period of 6 to 12 months in 17 of $20(85 \%)$ enrolled patients and in 17 of 19 (89\%) patients with drug-sensitive TB. Thirteen patients were cured of TB after 6 months of therapy, 3 after 7 months, and 1 at 10 months.

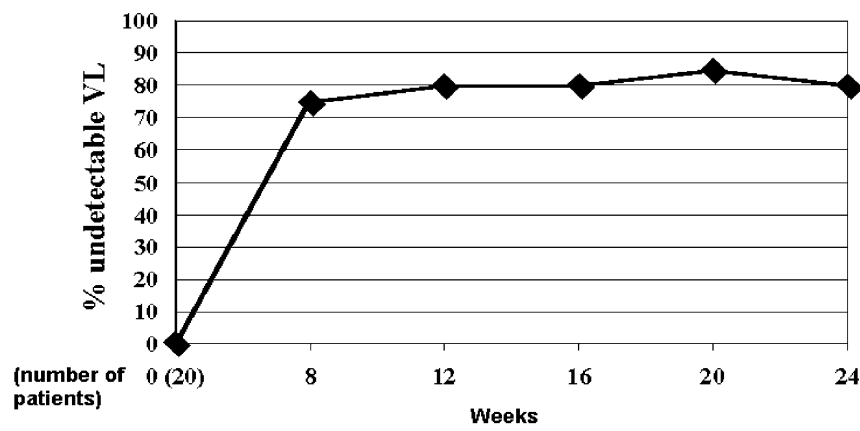

FIGURE 1. Percent of all enrolled patients with non-detectable viral load ( $<50$ copies).

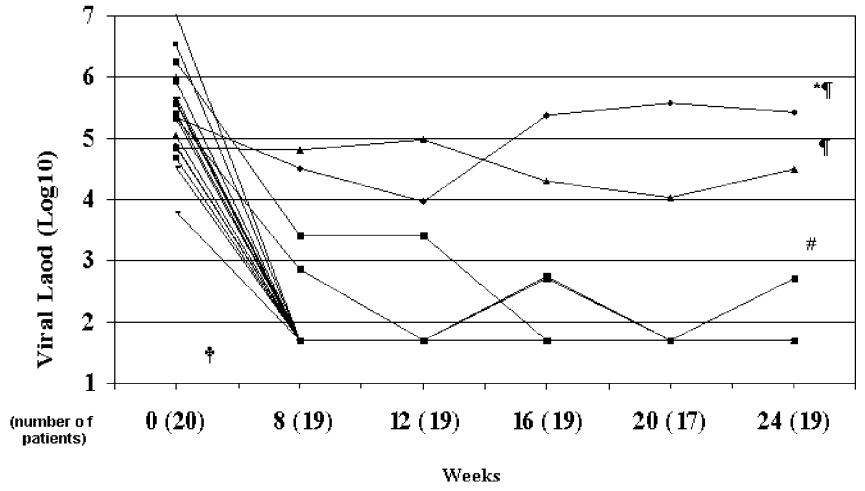

FIGURE 2. Twenty patients were enrolled: 19 remained on study and 17 completed standard TB and antiretroviral therapy and achieved a viral load $<50$ copies at 24 weeks. $\dagger$, withdrew at 2 weeks; *, unrecognized multidrug-resistant TB on enrollment; \#, poorly adherent, II, antiretroviral resistance.

HAART and TB medications were well tolerated. One patient withdrew after 2 weeks of therapy because of medication adverse effects, including loss of appetite, vomiting, diarrhea, abdominal pain, difficulty in sleeping, nightmares, and dizziness. In addition, social circumstances contributed to her discontinuation of therapy, because she was unable to disclose her HIV status to her employer and was therefore not able to come to the clinic on a daily basis. The most common medication adverse effects reported were dizziness and poor concentration in $8(40 \%)$ patients and insomnia in $2(10 \%)$ patients. These symptoms presented in the first few weeks after initiation of therapy and then subsided and were ascribed to efavirenz. Two patients transiently took off from work during this period as a result of these symptoms. Other reported symptoms were fatigue in $9(45 \%)$ patients, vomiting in $3(15 \%)$, abdominal pain and rash in $3(15 \%)$, and diarrhea in $2(10 \%)$. Apart from the patient noted previously, these symptoms were of grade 1 and 2 in severity and did not warrant discontinuation of HAART therapy.

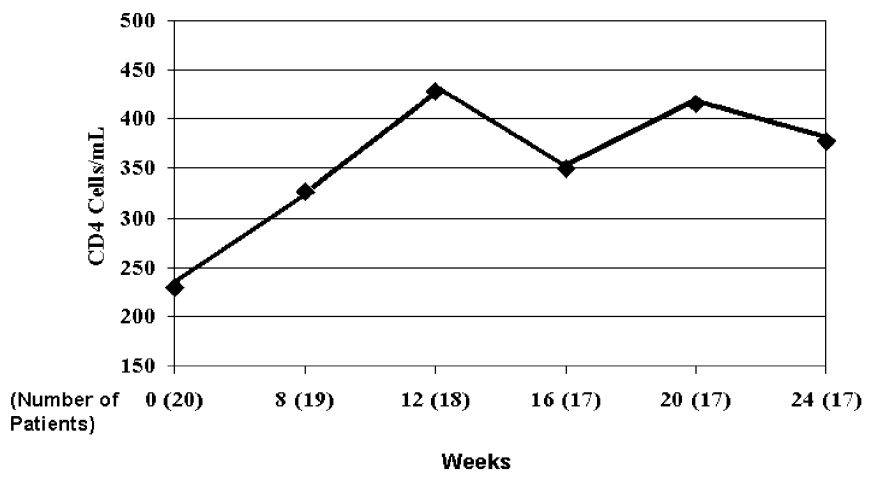

FIGURE 3. Change in mean CD4 cell count in patients on standard TB and antiretroviral therapy. 
The patients adhered well to the study requirements, missing only $5.5 \%$ of the 2078 weekday clinic visits and associated HAART and TB medication doses. The median number of missed visits was 1 per patient (range: 0-68 missed visits). The number of physician clinical monitoring visits per patient ranged between 9 and 14 over the entire study period. Among those patients who continued in the study, only 2 missed physician appointments. One missed 2 appointments after sustaining multiple fractures after a motor vehicle accident. A second patient who relocated during the course of the study continued taking TB medications but could not be located for approximately 3 months and was therefore not on HAART during this period. Apart from these 2 patients, all patients received weekend medication throughout the study and reported $100 \%$ weekend adherence. The study was well accepted by clinic staff and seemed to be well integrated into the clinic's procedures and practice.

At the time of study, the South African Rand (R) was approximately 11.00 to the US dollar and 10.00 to the Euro. The cost of treating pulmonary TB within the TB/DOT program in the Cyril Zulu Communicable Diseases Clinic was $\$ 345$ per patient for 6 months, including personnel, medications, chest radiography, and laboratory tests. The cost of incorporating HAART into the TB/DOT program during this period was $\$ 1286$ per patient, assuming a TB treatment duration of 6 months. Sixty percent of this cost, $\$ 777$ per patient, was related to the purchase of the antiretroviral drugs. Laboratory monitoring contributed $\$ 272$ per patient, and $\$ 237$ per patient was expended for additional study personnel costs.

\section{DISCUSSION}

This pilot study represents the first reported attempt to integrate HAART into an existing TB/DOT program in a resource-poor setting. The results of the study are encouraging and, although preliminary, support the feasibility and utility of this strategy. The study provides additional preliminary evidence regarding the efficacy and toxicity of a once-daily regimen of HAART in patients with HIV and TB. ${ }^{10-14}$

We have demonstrated that (1) it is feasible to introduce HAART into an existing TB/DOT program in a metropolitan clinic without disrupting or compromising clinic routines and practice, (2) a once-daily HAART regimen of efavirenz, 3TC, and ddI administered concomitantly with a standard rifampicin-based TB treatment regimen during TB/DOT is effective and well tolerated, (3) adherence to self-medication of HAART over weekends is excellent, (4) a high TB cure rate can be achieved, and (5) the additional cost of the strategy is largely related to the cost of antiretroviral therapy.

Rabkin et $\mathrm{al}^{13}$ have succinctly outlined the challenges and opportunities related to the provision of antiretroviral treatment in resource-poor settings. Further, it has been argued that structured implementation of HAART may prevent "antiretroviral anarchy" in sub-Saharan Africa. ${ }^{14}$ The high burden of disease caused by HIV and TB coinfection in South Africa and other resource-poor countries, the challenge of adherence to medication, ${ }^{8}$ and the widespread existence of the TB/DOT infrastructure recommend these programs as logical and appealing but previously untested sites for the structured introduction of antiretroviral therapy. The results of this pilot study suggest that introducing HAART through an existing TB/DOT program is a structured implementation that can be feasible, safe, effective, and acceptable to the patients and staff at these facilities.

This study also adds to the literature on therapeutic interventions that address the co-occurrence of HIV and TB. Studies from Brazil and South Africa suggest that the use of HAART in patients coinfected with HIV and TB but without active disease lowers the subsequent incidence of active TB. ${ }^{15,16}$ In most resource-poor settings, however, patients with HIV disease come to clinical attention with already active TB. In this pilot study, we have addressed this common scenario by employing directly observed therapy with both TB and HIV medications in an attempt to improve the outcome of the 2 diseases.

Directly observed therapy for TB was introduced more than 40 years ago as a method of ensuring medication adherence and treatment completion and decreasing the risk of drugresistant TB. This strategy has become a central component of the Directly Observed Therapies (DOTS) strategy recommended by the World Health Organization (WHO) for treatment of TB. ${ }^{17}$ More recently, successful DOT programs were noted to be those that supplemented observation of medication taking with several other components, including enhanced staff motivation and patient-centered supportive program elements. ${ }^{18}$ In the present study, medication administration under observation and social support were features of the design and likely contributed to its success.

Although the utility of administration of HAART by DOT has been questioned, ${ }^{19}$ the availability of potent oncedaily regimens makes this strategy more practical, particularly in the setting of an existing TB once-daily regimen. The availability of once-daily dosing of HAART has improved tolerability while maintaining efficacy and safety and is expected to increase adherence, reduce drug resistance, and improve clinical outcomes in the long term..$^{20,21}$ Modified communitybased HAART through DOT support has been successful in special settings in developed countries. ${ }^{22,23}$ Of relevance to the present study, this strategy of DOT HAART has also been successfully employed in a resource-poor setting in Haiti among patients with advanced HIV disease, although without active $\mathrm{TB}^{24}$

This study employed a once-daily regimen of ddI, 3TC, and efavirenz. This regimen was chosen based on medication availability in South Africa, known compatibility with rifampin, potency, and available literature on once-daily antiretroviral therapy regimens. ${ }^{10-12}$ This regimen has also been ad- 
ministered successfully during TB treatment in Brazil. ${ }^{25}$ This study is the first report of the coadministration of this oncedaily regimen with $\mathrm{TB}$ medications through an existing TB/DOT program, however.

Many physicians and experts currently recommend delay of HAART initiation in patients presenting with TB because of added pill burden and concern about drug interactions, toxicity, and risk of immune reconstitution events. In the present study, the added number of pills did not seem to compromise adherence to HAART during either concomitant administration with TB medication or weekend home selfadministration. Future once-daily regimens should result in a further decrease in the required number of pills. Dean et $\mathrm{al}^{26}$ demonstrated the effectiveness of concomitant TB and HAART treatment, although such therapy was associated with significant adverse events leading to interruptions in TB/HIV therapy. In our pilot study, medications were well tolerated, with only 1 patient withdrawing because of a combination of drug adverse effects and social issues. For 2 other patients, treatment interruptions were the result of environmental and social factors. Immune reconstitution events were not identified in this study, and the 1 patient who deteriorated clinically was found to have multidrug-resistant TB.

Overall, in this pilot feasibility study, 17 of 19 (89\%) patients with drug-sensitive TB receiving standard therapy were cured. The WHO has reported an average worldwide TB treatment success rate of $82 \%$ for the year 2000 utilizing a DOTS strategy. Treatment success was substantially below average in the African region (72\%), however. ${ }^{27}$ In South Africa, these rates have been variable, ranging from $54 \%$ to $89 \%$ in published studies. ${ }^{28,29}$ The TB cure rate in the province of KwaZulu Natal in 1999 was $40 \%,{ }^{30}$ and data for 2001 for the clinic where this study was conducted showed a cure rate of $66.5 \%$ for new smear-positive TB (personal communication, Dr. Surie Chinappa, 2003). Although the number of participants in the present pilot study was small and carefully selected, the high TB cure rate suggests the possibility of another potential benefit for treating both diseases together in this setting.

HAART is considered a high-cost intervention in the care of HIV disease in resource-constrained settings. Wilkinson et $\mathrm{al}^{31}$ estimated that it costs approximately $\$ 345$ to cure a patient with TB in South Africa. The major added cost associated with the addition of HAART to TB/DOT in this study was that of antiretroviral medications. Thus, overall costs of the strategy can be reduced substantially if costs of HAART can be further reduced. Procurement and administration of less costly HAART regimens in resource-limited settings remain major challenges for this strategy and for the introduction of antiretroviral therapy in general. Laboratory monitoring added additional cost, but the frequency of CD4 cell counts and viral load testing could be substantially reduced as is recommended for clinical and public health practice. ${ }^{32}$
Although the results of this pilot study are encouraging, several limitations should be recognized. The study included a small number of patients and was carried out at a single site and patients were ambulatory and selected to meet study entry criteria, thereby potentially limiting the generalizability of the findings. In addition, despite careful monitoring for adverse effects and toxicities, the small size of the study may have limited the ability to detect important toxicities. Similarly, although the study seemed to be accommodated well within usual clinic activities, the small number of subjects, presence of research staff, and absence of a formal analysis of clinic functions limit this observation. Finally, the strategy failed in 2 patients who developed antiretroviral resistance. This may be related to subtherapeutic drug levels resulting from decreased medication absorption or increased rifampicininduced metabolism of efavirenz. The correct dose of efavirenz for administration with rifampicin remains controversial. Most recent recommendations favor maintaining the standard 600 -mg dose, ${ }^{12}$ although others recommend increasing the dose to $800 \mathrm{mg}$ administered once daily. ${ }^{30}$ Other larger series have successfully used the lower dose. ${ }^{25}$ The high frequency of early efavirenz-induced adverse effects experienced by participants in the present study suggests that using a higher dose might be associated with an unacceptable adverse effect profile and hazard to some patients. Further studies of the pharmacokinetics of various doses of efavirenz in the presence of rifampicin are needed to resolve this controversy.

We recognize that the coadministration of HAART and TB therapy through the existing TB/DOT program does not fully address the more long-term requirement for antiretroviral therapy administration. This does not obviate the utility of the strategy, however. We view this strategy as providing an entry point for identification of eligible patients for HIV treatment and initiation of HAART and the provision of an initial successful structured experience with antiretrovirals, the benefits of which may extend well into the subsequent period of longterm self-administration.

The results of this pilot study warrant full and critical evaluation in a large randomized trial of the short- and longterm benefits and risks of concurrent versus deferred HAART in coinfected patients treated within TB/DOT programs. Such a trial is currently in development. ${ }^{33}$ In the meantime, given the urgency of providing treatment of HIV disease in resourcepoor settings, the feasibility and utility of this strategy should be confirmed at other sites and, if reproducible, should be strongly considered for wider expansion.

\section{ACKNOWLEDGMENTS}

The authors acknowledge the contributions of study nurse Colleen Ziyane, study coordinator Vikesh Naidoo, the staff at the Cyril Zulu Communicable Diseases Clinic, Marita Murrman, the patients who participated in the study, and the 
Department of Chemical Pathology, Nelson R. Mandela School of Medicine, University of Kwa Zulu Natal.

\section{REFERENCES}

1. Abdool Karim Q, Abdool Karim SS. South Africa: host to a new and emerging HIV epidemic. Sex Transm Infect. 1999;75:139-147.

2. Wilkinson D, Davies GR. The increasing burden of TB in rural South Africa-impact of the HIV epidemic. S Afr Med J. 1997;87:447-450.

3. Palella FJ Jr, Delaney KM, Moorman AC, et al. Declining morbidity and mortality among patients with advanced human immunodeficiency virus infection. HIV Outpatient Study. N Engl J Med. 1998;338:853-860.

4. De Cock KM, Lucas SB, Lucas S, et al. Clinical research, prophylaxis, therapy, and care for HIV disease in Africa. Am J Public Health. 1993; 83:1485-1489.

5. Burman WJ, Jones BE. Treatment of HIV-related tuberculosis in the era of effective antiretroviral therapy. Am J Respir Crit Care Med. 2001;164: $7-12$.

6. Schulger NW. Issues in the treatment of active TB in HIV infected patients. Clin Infect Dis. 1999;28:130-135.

7. Narita M, Ashkin D, Hollender ES, et al. Paradoxical worsening of tuberculosis following antiretroviral therapy in patients with AIDS. Am J Respir Crit Care Med. 1998;158:157-161.

8. Mannheimer S, Friedland G, Matts J, et al. Antiretroviral adherence and its consistency predicts outcomes in HIV clinical trials. Clin Infect Dis. 2002;34:1115-1121.

9. The South African Tuberculosis Control Programme Practical Guidelines Ministry of Health. 4th ed. Pretoria, 2000. Available at: http://www. doh.gov.za/tb/index.

10. Landman R, Schieman R, Thiam S, et al. Evaluation at 6 months of a once a day HAART regimen in treatment naïve HIV 1 infected adults in Senegal (ANRS 12-04 Study) [abstract 491]. Presented at the Eighth Conference on Retrovirus and Opportunistic Infections, Chicago, February 2001.

11. Maggiolo F, Migliorino M, Maserati R, et al. Virological and immunological responses to once-a-day antiretroviral regimen with didanosine, lamivudine and efavirenz. Antivir Ther. 2001;6:249-253.

12. Updated guidelines for the use of rifabutin and rifampicin for the treatment and prevention of tuberculosis among HIV infected patients taking protease inhibitors or non-nucleoside reverse transcriptase inhibitors. MMWR. 2000;49:185-189.

13. Rabkin M, El-Sadr W, Katzenstein DA, et al. Antiretroviral treatment in resource-poor settings: clinical research priorities. Lancet. 2002;360: $1503-1505$.

14. Harries AD, Nyangulu DS, Hargreaves NJ, et al. Preventing antiretroviral anarchy in sub-Saharan Africa. Lancet. 2001;358:410-414.

15. Santoro-Lopes G, de Pinho AM, Harrison LH, et al. Reduced risk of tuberculosis among Brazilian patients with advanced human immunodeficiency virus infection treated with highly active antiretroviral therapy. Clin Infect Dis. 2002;34:543-546.

16. Badri M, Wilson D, Wood R. Effect of highly active antiretroviral therapy in the incidence of tuberculosis in South Africa: a cohort study. Lancet. 2002;359:2059-2064.

17. Maher D, Chaulet P, Spinaci S, et al. Treatment of Tuberculosis: Guidelines for National Programmes. 2nd ed. Geneva: WHO; 1997.

18. Volmink J, Matchaba P, Garner P. Directly observed therapy and treatment adherence. Lancet. 2000;355:1345-1350.

19. Lucas GM, Flexner CW, Moore RD. Directly administered antiretroviral therapy in the treatment of HIV infection: benefit or burden? AIDS Patient Care STDS. 2002;16:527-535.

20. Rosenbach KA, Allison R, Nadler JP. Daily dosing of highly active antiretroviral therapy. Clin Infect Dis. 2002;34:686-692.

21. Ena J, Pasuau F. Once-a-day highly active antiretroviral therapy: a systematic review. Clin Infect Dis. 2003;36:1186-1190.

22. Mitty JA, Stone VE, Sands M, et al. Directly observed therapy for the treatment of people with human immunodeficiency virus infection: a work in progress. Clin Infect Dis. 2002;34:984-990.

23. Altice FL, Mezger J, Hodges J, et al. Directly observed therapy for HIV+ drug users. Presented at the Second IAS Conference on HIV Pathogenesis and Treatment, Paris, July 2003.

24. Farmer P, Leandre F, Mukherjee J, et al. Community-based treatment of advanced HIV disease: introducing DOT-HAART (directly observed therapy with highly active antiretroviral therapy). Bull World Health Organ. 2001;9:1145-1151.

25. Pedral-Samapio D, Alves C, Netto E, et al. Efficacy of efavirenz $600 \mathrm{mg}$ dose in the ARV therapy regimen for HIV patients receiving rifampicin in the treatment of tuberculosis [abstract 784]. Presented at the 10th Conference on Retroviruses and Opportunistic Infections, Boston, January 2003.

26. Dean GL, Edwards SG, Ives NJ, et al. Treatment of tuberculosis in HIVinfected persons in the era of highly active antiretroviral therapy. AIDS. $2002 ; 16: 75-83$.

27. WHO Report. Global Tuberculosis Control. WHO/CDS/TB/2003. Geneva: WHO; 2003:316.

28. Zwarenstein M, Schoeman JH, Vundule C, et al. Randomised controlled trial of self-supervised and directly observed treatment of tuberculosis. Lancet. 1998;352:1340-1343.

29. Wilkinson D. High compliance tuberculosis treatment programme in a rural community. Lancet. 1994;343:647-648.

30. Hoque AKM, Meidany F, Marlani D, et al. KwaZulu-Natal Epidemiology Bulletin. KwaZulu-Natal Department of Health; Pretermaritzburg 2002.

31. Wilkinson D, Floyd K, Gilks CF. Costs and cost-effectiveness of alternative tuberculosis management strategies in South Africa-implications for policy. S Afr Med J. 1997;87:451-455.

32. WHO. Scaling Up Antiretroviral Therapy in Resource-Limited Settings. Guidelines for a Public Health Approach. WHO; Geneva 2002.

33. START. Starting Tuberculosis and Anti-Retroviral Therapy, A Randomized Controlled Trial to Assess the Effect of Integrated Tuberculosis and HIV Care on the Incidence of AIDS-Defining Conditions and Mortality in Participants Co-Infected with Tuberculosis and HIV. CAPRISA, Division of AIDS, NIAID, NIH. University of Kwa Zulu Natal, South Africa. Bethesda, MD 2004 